\title{
Instillation Negative Pressure Wound Therapy: An Effective Tool for Complex Spine Wounds
}

\author{
Julie M. West, ${ }^{1, *}$ Sumanas W. Jordan, ${ }^{1}$ Ehud Mendel, ${ }^{2,3}$ \\ Safdar N. Khan, Rajiv Y. Chandawarkar, ${ }^{1,3}$ and lan L. Valerio ${ }^{1,3}$ \\ Departments of ${ }^{1}$ Plastic Surgery, ${ }^{2}$ Neurosurgery and ${ }^{3}$ Orthopedic Surgery, The Ohio State University Wexner \\ Medical Center, Columbus, Ohio.
}

Objective: Infection prevention in spinal surgeries involving implantation of hardware is of utmost priority. Furthermore, successful eradication of infection in hardware salvage is likewise critical in maintaining the long-term retention of the spinal hardware construct.

Approach: We report a retrospective case series of three cases where the utilization of a VAC with instillation (VACi) in conjunction with surgical debridement aided in infection control and eradication for both preimplantation and hardware salvage spine patients.

Results: Three patients were included. In case 1, VACi was utilized in conjunction with surgical debridement and IV antibiotics in the setting of acute preoperative infection to eradicate infection and enable necessary spinal hardware implementation. Cases 2 and 3 are representative of VACi for salvage of exposed spinal hardware in both the early and delayed infection presentation settings. In both cases, patients developed postoperative infections following spinal instrumentation VACi was utilized in conjunction with surgical debridement and IV antibiotics. Hardware removal was avoided in both cases. All three patients healed completely without residual evidence of infection.

Innovation: VACi showed its effectiveness in timely infection eradication before spinal hardware instrumentation and with postoperative spine hardware salvage.

Conclusion: This case series demonstrates that VACi can provide infection eradication both preoperatively in high-risk surgical sites, facilitating necessary hardware implementation and postoperatively in situations of hardware salvage.

Keywords: negative pressure, VAC with instillation, debridement, infection

\section{INTRODUCTION}

WHEN IMPLANTING A FOREIGN body such as hardware, preventing infection is an utmost priority. Preoperative patient goals include a stable wound bed, control of preexisting infection and inflammation to the extent possible, and systemic medical optimization of chronic medical problems and nutritional deficiencies. Routine intraoperative techniques to decrease bacterial contamination and minimize infection risk may include strict sterile field preparation, antibiotic irrigation, debridement, and use of antibiotic powders and beads. ${ }^{1,2}$ Even under ideal conditions, low risk patients may develop hardware infections

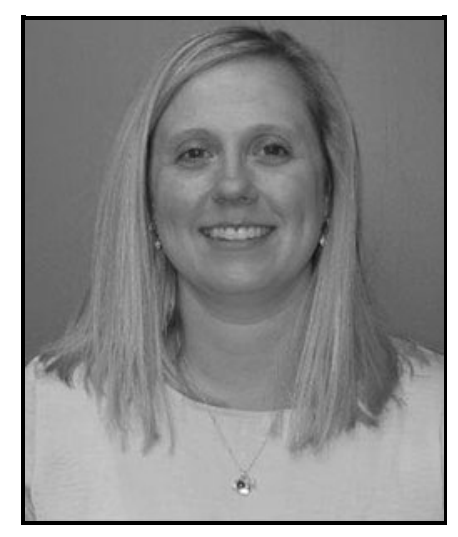

Julie M. West, MS, PA-C

Submitted for publication March 14, 2018. Accepted in revised form April 12, 2018.

${ }^{*}$ Correspondence: Department of Plastic Surgery, The Ohio State University Wexner Medical Center, 915 Olentangy River Road, Suite 2100, Columbus, $\mathrm{OH} 43212$

(e-mail: Julie.West@osumc.edu) 
postoperatively that require hospital readmission, long-term intravenous antibiotics, and reoperation. In some cases, biofilm contamination prevents clearance of the infection and hardware removal is necessary. When faced with an actively infected patient or a patient with an open wound preoperatively, surgeons are hesitant to place hardware due to the high risk of adverse outcomes, specifically infection. There is no clear consensus on how to treat surgical site infection following spinal instrumentation. Surgical debridement is advocated across the board, but indications on implant retention versus removal and duration of antibiotic therapy remain unclear. ${ }^{3}$

\section{CLINICAL PROBLEM ADDRESSED}

Herein, we report the utilization of VAC Veraflo and VAC Veraflo Cleanse Choice therapy in conjunction with surgical debridement. Case 1 describes treatment in the setting of acute preoperative infection to eradicate infection and enable necessary spinal hardware implementation. Cases 2 and 3 are representative of salvage of exposed spinal hardware in both the early and delayed infection settings. We have performed three cases of spinal hardware salvage.

\section{MATERIALS AND METHODS}

This is a retrospective review of three cases treated at a single institution. Patients were included in the case series if they presented with either an actively infected back wound requiring immediate hardware instrumentation, or an infected back wound with existing spinal hardware where successful hardware salvage was in the patient's best interest.

\section{Case 1}

A 66 year-old male with a past medical history significant for uncontrolled insulin-dependent diabetes with a hemoglobin A1C of 9 and renal cell carcinoma requiring right total nephrectomy with known pulmonary nodules presented with an open fungating thoracic back wound. Two months before presentation to our clinic, he had developed new-onset thoracic back pain without neurologic symptoms and was subsequently diagnosed with a soft tissue mass in this region. He had undergone partial resection of the mass complicated by continued purulent drainage despite multiple attempts at debridement. Magnetic resonance imaging $(\mathrm{MRI})$ revealed a $2.4 \times 8.0 \times 9.0 \mathrm{~cm}$ paraspinous mass and an enhancing lesion at T6 with cord compression (Fig. 1a), at which point he was transferred to our institution.
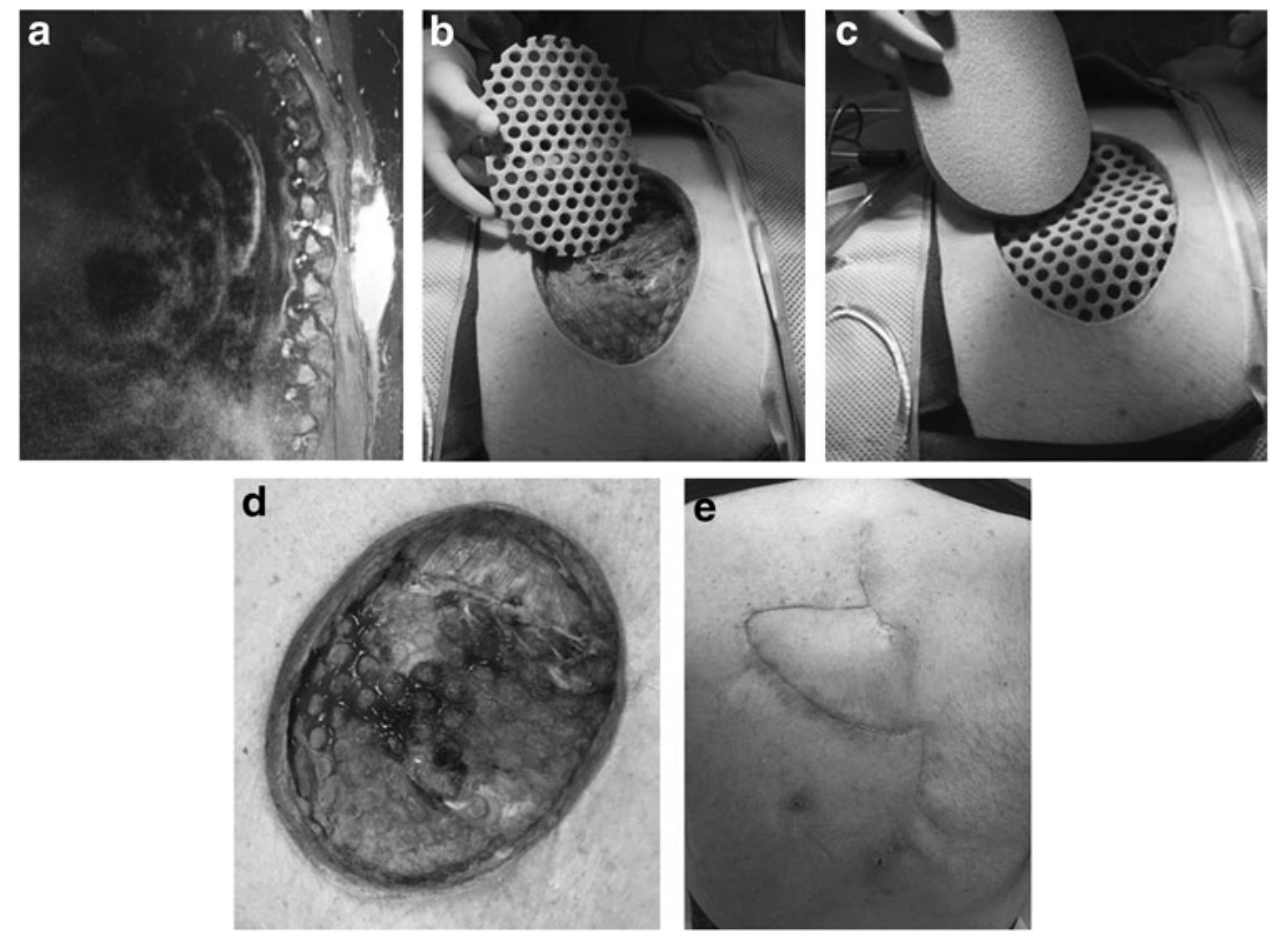

Figure 1. Case 1 (a) preoperative MRI showing $2.4 \times 8.0 \times 9.0 \mathrm{~cm}$ paraspinous mass, (b) postdebridement application of Veraflo Cleanse Choice dressing, contact layer (c) postdebridement application of Veraflo Cleanse Choice dressing, second layer, (d) preclosure VAC removal, (e) 6 week postoperative followup. MRI, magnetic resonance imaging. 
He presented with an $8 \times 10 \mathrm{~cm}$ subcutaneous left upper back defect with a $1.5 \mathrm{~cm}$ open incisional wound that drained thick brown purulent material. After a multidisciplinary team evaluation, neurosurgery and surgical oncology planned for a staged resection of the mass. This would be followed by hardware instrumentation for spinal stability and soft tissue reconstruction by plastic surgery once the infection was eradicated and the wound was stable. Following a gross en bloc resection of the $12 \times 14 \mathrm{~cm}$ back mass, an open wound with exposed spinous processes at multiple thoracic levels remained. The VAC Veraflo Cleanse Choice dressing was placed intraoperatively and set to $125 \mathrm{mmHg}$ continuous suction and Dakin's $0.125 \%$ solution was instilled for $10 \mathrm{~min}$ at a time in $3 \mathrm{~h}$ intervals to facilitate the removal of infectious material and cleanse the wound postdebridement (Fig. 1b-d). Wound cultures grew Staphylococcus epidermidis and pathology confirmed metastatic renal cell carcinoma. Infectious disease was consulted and recommended intravenous ertapenem daily for 6 weeks.

Following the initial VAC Veraflo Cleanse Choice placement after tumor resection, the patient returned to the operating room twice for weekly wound assessment, debridement, postdebridement cultures, and instillation dressing change. At the second dressing change, the wound showed evidence of healing, and postdebridement wound cultures were negative. On day 17 from initial resection, the patient underwent vertebral decompression and spinal fusion along with bilateral paraspinous muscle flaps and a right trapezius flap closure. The patient completed his course of antibiotics, postoperative rehabilitation program, and subsequently healed without adverse sequelae (Fig. 1e).

\section{Case 2}

A 51-year-old male with a past medical history significant for insulin-dependent diabetes, and recurrent L5-S2 sacral chordoma who had undergone radiation, and three resections with surgical hardware instrumentation and flap closure presented with a 1 week history of a 2.5 -cm draining lumbar back wound. Wound cultures obtained upon hospital admission grew methicillin-resistant Staphylococcus aureus (MRSA), and CT and MRI revealed tumor recurrence and a $2.9 \times 1.2 \mathrm{~cm}$ fluid collection at L3 (Fig. 2a). There were no further surgical recommendations for the patient in terms of his tumor. To address the infection, the patient was presented with two options: (1) attempt to
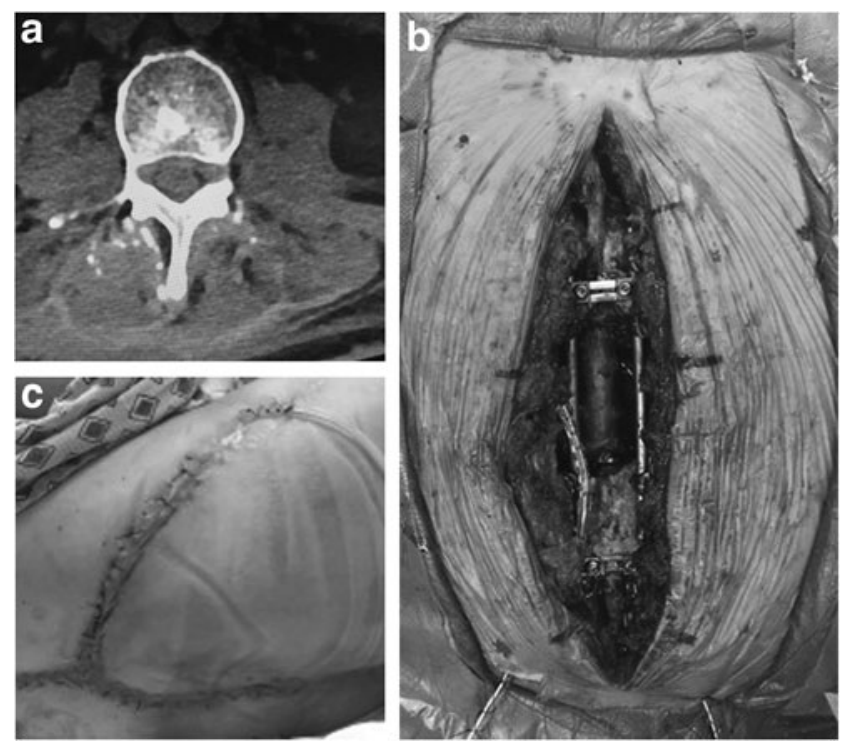

Figure 2. Case 2 (a) preoperative CT demonstrating a $2.9 \times 1.2 \mathrm{~cm}$ fluid collection at L3, (b) postdebridement wound photo, (c) 4 week postoperative follow-up.

salvage the hardware with multiple washouts, instillation VAC placement, and culture-guided antibiotic therapy, or (2) have the hardware removed. At that time, he was functionally independent and removing his hardware could have a devastating effect on the patient's mobility and his bowel and bladder control. The patient elected to try and salvage his hardware.

The patient was taken to the operating room and the neurosurgery team examined his hardware and tightened the screws. Following this, the plastic surgery team sharply debrided the wound and irrigated with $3 \mathrm{~L}$ of normal saline containing triple antibiotic solution of ancef, bacitracin, and gentamycin. A $15 \times 6 \times 4-\mathrm{cm}$ lumbar spine wound with exposed hardware remained (Fig. 2b). The VAC Veraflo Cleanse Choice dressing was placed intraoperatively and set to $125 \mathrm{mmHg}$ continuous suction and Dakin's 0.125\% solution was instilled for $10 \mathrm{~min}$ durations in $3 \mathrm{~h}$ intervals to facilitate the removal of infectious material and cleanse the wound. Postdebridement wound cultures again grew MRSA. Infectious disease was consulted and recommended intravenous vancomycin and oral rifampin daily for 6 weeks followed by chronic suppression with doxycycline. On postoperative day 4 , the patient returned for repeat debridement and culture at which time the decision was made to partially close the deep layers of the wound and reapply an instillation VAC Veraflo dressing while his cultures finalized. Postdebridement 
wound cultures again grew MRSA. On postoperative day 6 he returned to the operating room for another debridement and instillation VAC placement. Postdebridement cultures were negative, and on postoperative day 11 from initial debridement, the patient underwent closure with right gluteal advancement flap, paraspinous muscle flaps, and antibiotic bead placement. The patient subsequently completed his 6-week course of intravenous antibiotics and began chronic suppressive antibiotics (Fig. 2c). He was last examined at 15 month follow-up, and was well-healed without wound breakdown or evidence of infection related to spinal hardware.

\section{Case 3}

A 19-year-old female with a past medical history significant for obesity with a body mass index of 49 sustained an orthopedic polytrauma and underwent fixation of thoracic and lumbar spine in addition to open reduction internal fixation and screw fixation of the pelvis. At the time of her spinal hardware instrumentation, plastic surgery was consulted intraoperatively for assistance with soft tissue coverage. The patient underwent dead space obliteration and flap closure with bilateral paraspinous muscle flaps. Three and a half weeks postoperatively, the patient developed purulent drainage from her spine incision and in her drains bulbs and was taken to the operating room for debridement. After sharp debridement of necrotic tissue and copious irrigation with dilute betadine solution, the thoracic wound measured $10 \times 6 \times 4 \mathrm{~cm}$ with exposed hardware. The VAC Veraflo dressing was placed intraoperatively and set to $125 \mathrm{mmHg}$ continuous suction and Dakin's $0.125 \%$ solution was instilled for 10 min durations in $3 \mathrm{~h}$ intervals to facilitate the removal of infectious material and to cleanse the wound. Wound cultures grew methicillin-resistant $S$. epidermidis, Prevotella, Proteus, Enterococcus faecalis, and Candida albicans. Infectious disease was consulted and recommended intravenous ertapenem, daptomycin, and oral fluconazole for 8-12 weeks. On postoperative days 5,7 , and 9 the patient underwent instillation VAC changes at bedside. The wound was clean with a granulating base and on postoperative day 7 the Dakin's solution was discontinued and normal saline was instilled for $10 \mathrm{~min}$ duration in $3 \mathrm{~h}$ intervals. On postoperative day 14, the patient underwent washout and debridement of the thoracic back wound with left trapezius and left paraspinous muscle flaps for closure. The patient completed her antibiotic regimen per the Infectious Disease ser- vice, and when last examined at 6 month follow-up she remained well healed without evidence of infection.

\section{RESULTS}

Three patients were included in our case series and all went on to complete healing with hardware in place. In case 1 , VAC with instillation (VACi) was utilized in conjunction with surgical debridement and IV antibiotics in the setting of acute preoperative infection to eradicate infection and enable necessary spinal hardware implementation. Cases 2 and 3 are representative of VACi for salvage of exposed spinal hardware in both the early and delayed infection presentation settings. In both cases, patients developed postoperative infections following spinal instrumentation VACi was utilized in conjunction with surgical debridement and IV antibiotics. From time of initial presentation all patients were treated and closed within 17 days and healed completely without residual evidence of infection.

\section{DISCUSSION}

Negative pressure wound therapy (NPWT) revolutionized the management of acute and chronic wounds. ${ }^{4,5}$ When placed on an appropriately prepared wound, NPWT promotes healing, microdebridement, the formation of granulation tissue, and angiogenesis. ${ }^{4-7}$ NPWT also assists with removal of bacterial debris, edema, excess inflammatory factors, and the approximation of wound edges, creating a suitable climate for wound healing. ${ }^{4-7}$ VACi incorporates the concept of NPWT with the additional cyclical delivery of solutions that are cleansing and antimicrobial in nature. ${ }^{8}$ Lehner et al. describe this cycle in three phases: the instillation phase, in which the solution is administered to the wound bed; hold phase, in which the solution is in contact with the wound without application of sub-atmospheric pressure; and vacuum phase, in which negative pressure is reapplied. ${ }^{8}$

In a retrospective cohort study comparing traditional NPWT and VACi in patients with infected wounds requiring operative debridement, patients receiving $\mathrm{VACi}$ had statistically significant decreased hospital length of stay, decreased operations, and decreased length to final surgical procedure compared to patients who received NPWT alone. ${ }^{9}$ Furthermore, in the management of acute and chronic infections of orthopedic hardware, in which the primary goal was infection eradication and hardware retention, Lehner et al. demonstrated 
$86 \%$ of acute and $80 \%$ of chronic infections at 4-6 months follow-up had successful implant retention when managed with VACi. ${ }^{8}$ While this study was observational in nature, it documents adequate antimicrobial management of complex wounds in the setting of hardware that are often complicated by biofilm formation.

Literature has revealed that VACi can be used in the management of acute and chronic osteomyelitis, infected hardware, and other complicated wound closures.

\section{KEY FINDINGS}

- VACi can be used in conjunction with surgical debridement and antibiotic therapy to prepare a complex wound with hardware for closure in a short amount of time.

- VACi can help more rapidly clear infection with its continuous cyclical delivery of irrigation thus allowing hardware to be placed successfully.

- Further research is needed to determine the role VACi can play in clearing infection preoperatively before hardware placement or in hardware salvage in other areas of the body.
Our case series demonstrates that VACi

can provide infection eradication both preoperatively in high-risk surgical sites, facilitating necessary hardware implementation and postoperatively in situations of hardware salvage. Dakin's $0.125 \%$ solution was instilled via the VACi after debridement because of its microbicidal properties and its ability to penetrate residual biofilm. Though it can cause damage to tissues long term, we were more concerned about eradicating infection before closure and this was a successful solution to instill regularly to help achieve that goal. Further studies are necessary to better develop infection reducing protocols using the VAC Veraflo and Veraflo Cleanse Choice dressings to facilitate successful hardware instrumentation or salvage.

\section{INNOVATION}

The VAC Veraflo and Veraflo Cleanse Choice dressings helped eradicate residual infection and prepare wound beds for closure. Our cases highlight the fact that while instrumenting hardware in an open and infected area is high risk, it is sometimes medically necessary. Second, it shows that with cautious optimism hardware salvage in a patient without further surgical options is possible. They serve as a basis for further research into the role that VACi can play in clearing infection preoperatively before hardware placement or in hardware salvage in other areas of the body.

\section{ACKNOWLEDGMENTS AND FUNDING SOURCES}

No funding was given or requested for this article.

\section{AUTHOR DISCLOSURE AND GHOSTWRITING}

No competing financial interests exist. The content of this article was expressly written by the authors listed. No ghostwriters were used to write this article.

\section{ABOUT THE AUTHORS}

Julie M. West, MS, PA-C, is a Physician Assistant in plastic and reconstructive surgery at The Ohio State University Wexner Medical Center who has extensive experience with wound care and clinical trials. Sumanas W. Jordan, MD, PhD, is currently a microsurgery fellow at Ohio State. She has an interest in medical innovations and reconstructive surgery. Ehud Mendel, MD, is the clinical director of Ohio State's Spinal Biodynamics and Ergonomics Laboratory, and a professor of neurosurgery, oncology, orthopedics, and integrated engineering. His area of speciality is in spinal tumors. Safdar N. Khan, MD, is the director of the Division of Spine in the Department of Orthopedics at Ohio State who has both strong clinical and research focuses in his practice. Rajiv Y. Chandawarkar, MD, MBBS, is a plastic and reconstructive surgeon at Ohio State and the director of Ohio State's Limb Preservation Program. Ian L. Valerio, MD, is an accomplished surgeon who served 4 years as a reconstructive surgeon in the U.S. Navy at trauma centers across the world before joining The Ohio State University Wexner Medical Center. His research interests include reconstructing and salvaging parts of the human body after trauma or disease, advancing stem-cell therapies, and improving prosthetics to improve people's lives. 


\section{REFERENCES}

1. Kapadia BH, Berg RA, Daley J, Fritz JA, Bhave A, Mont MA. Periprosthetic joint infection. Lancet 2016;387:386-394.

2. McConoughey SJ, Howlin R, Granger JF, et al. Biofilms in periprosthetic orthopedic infections. Future Microbiol 2014;9:987-1007.

3. Lall RL, Wong AP, Lall RR, Lawton CD, Smith ZA, Dahdaleh NS. Evidence-based management of deep wound infection after spinal instrumentation. J Clin Neurosci 2015;22:238-242.

4. Orgill DP, Bayer LR. Negative pressure wound therapy: past, present and future. Int Wound $J$ 2013;10(Suppl 1):15-19.

5. Negosanti L, Sgarzani R, Nejad P, et al. VAC therapy for wound management in patients with contraindications to surgical treatment. Dermato Ther 2012;25:277-280.

6. Buck DW, Galiano RD. Chapter 3: wound care. In: Grabb and Smith's Plastic Surgery, 7th ed. Philadelphia, PA: Lippincott Williams and Wilkins, 2014 20-28.

7. Gage MJ, Yoon RS, Egol KA, Liporace FA. Uses of negative pressure wound therapy in orthopedic trauma. Orthop Clin North Am 2015;46: 227-234.

8. Lehner B, Fleischmann W, Becker R, Jukema GN First experiences with negative pressure wound therapy and instillation in the treatment of infected orthopaedic implants: a clinical observational study. Int Orthop 2011;25:1415-1420.
9. Kim PJ, Attinger CE, Steinberg JS, et al. The impact of negative-pressure wound therapy with instillation compared with standard negativepressure wound therapy: a retrospective, historical cohort, controlled study. Plast Reconstr Surg 2014; 133:709-716.

\section{Abbreviations and Acronyms}

$\mathrm{MRI}=$ magnetic resonance imaging

MRSA = methicillin-resistant Staphylococcus aureus

NPWT $=$ negative pressure wound therapy

$\mathrm{VACi}=\mathrm{VAC}$ with instillation 